EXTENDED REPORT

\title{
Effect of age on adult stereoacuity as measured by different types of stereotest
}

\section{Garnham, J J Sloper}

Br J Ophthalmol 2006;90:91-95. doi: 10.1136/bjo.2005.077719

See end of article for authors' affiliations .....................

Correspondence to: Mr John Sloper Moorfields Eye Hospital, City Road, London ECIV 2PD, UK; john.sloper@ dial.pipex.com

Accepted for publication 4 August 2005
Aim: To examine how stereoacuity changes with age as measured by a variety of stereotests. Methods: Stereoacuity has been measured in 60 normal subjects aged 17-83 years by a single observer using TNO, Titmus, Frisby near, and Frisby-Davis distance stereotests. Motor fusion was measured at $1 / 3$ metre and 6 metres.

Results: Overall stereoacuity measured by all tests showed a mild decline with age $(p<0.001$ for all tests; Spearman rank correlation). A marked reduction to screening or absent levels of stereoacuity was seen in five subjects aged over 55, but only with the TNO stereotest. All these subjects were able to achieve a stereoacuity of 200 seconds of arc or better with the Titmus test and 340 seconds of arc or better using the Frisby near stereotest. There was a small decline with age in the base out motor fusion range measured at 6 metres ( $p<0.05$; Spearman rank correlation). No subject described difficulty in judging distances for everyday tasks.

Conclusions: Although subjects showed some decline in stereoacuity with age by all tests, the large drop in stereoacuity seen in some older subjects using the TNO test was probably due to difficulty overcoming the dissociative effect of the test rather than a true reduction in cortical disparity detection. Results of random dot stereotests should be interpreted with caution in older patients, particularly with respect to their ability to perform everyday visual tasks.
A number of studies have described reduced stereoscopic function in older people, ${ }^{1-10}$ with one study describing a "catastrophic" drop in stereoacuity in some subjects over the age of 60 years. ${ }^{10} \mathrm{~A}$ high incidence of deficient stereopsis has been described in population studies of elderly subjects, ${ }^{37}$ but this was often associated with poor vision in one eye. These studies have not generally measured stereopsis across the full adult age range in normal subjects and most have only used one stereotest, commonly with random dot stimuli.

In a recent study of the effects of a unilateral, idiopathic macular hole on stereoacuity it became apparent that the TNO random dot test was failing to detect levels of stereoscopic function which were demonstrable by the contour based Titmus test, due care being taken to ensure that monocular clues were not being used. ${ }^{11}$ Although stereotests that use random dot stimuli are generally regarded as the "gold standard" for measuring stereopsis, because they contain no monocular clues and so avoid false positive errors, it is also important to avoid false negative errors when assessing stereoscopic function.

A battery of stereotests that use different types and sizes of stimuli has therefore been used to study stereoacuity in a large group of normal subjects who ranged in age from young adults to the elderly. This would appear to be the first study in which stereoacuity has been measured over the whole adult age range by a single observer using a variety of stereotests.

\section{SUBJECTS AND METHODS}

Stereoacuity has been measured in 60 normal subjects aged 17-83 years. The majority of subjects were recruited during their attendance at a lacrimal clinic, with most of the remainder being non-medical or non-orthoptic members of staff. No subject had any history of cognitive impairment. All subjects underwent an orthoptic examination including measurement of Snellen acuity at 6 metres, reduced Snellen acuity at $1 / 3$ metre and motor fusion for near and distance using prisms. All had a best corrected distance Snellen acuity of at least 6/9 in each eye, wore their appropriate refractive correction for testing at distance and near, and had no known ophthalmic disease. Subjects were asked whether they had any problems with judging distances for everyday tasks such as pouring a drink into a cup.

Stereoacuity was measured by the same experienced orthoptist using TNO, Titmus, Frisby near, and Frisby-Davis distance stereotests. Test order was varied between patients.

The TNO test (Laméris Ootech BV, Nieuwegein) uses random dot stimuli with red-green glasses to separate the images presented to each eye. There are no monocular clues and the stereotarget is not outlined by monocularly visible contours. It is the most dissociative of the stereotests used and has the smallest stimulus elements.

The Titmus test (Stereo Optical, Chicago, IL, USA) uses black, contoured stimuli, with Polaroid glasses to separate the stimuli presented to each eye. Clues which allow the circle with disparity to be identified as different under monocular viewing conditions are present, but in a recent study in children such monocular clues were not found to be a significant problem at stereoacuities of 160 seconds of arc or better. ${ }^{12}$ The use of monocular clues can be avoided in cooperative adults by ensuring that the subject is truly seeing the target circle standing out from the page and by checking that, when the page is inverted to reverse the disparity, the subject sees the target circle behind, rather than in front of the other circles.

The Frisby near stereotest uses real depth targets. Recognition of the stereo stimulus depends on detecting a virtual contour delineated by a group of these elements. No distinguishable contour defining the stereotarget exists before fusion. Monocular parallax clues are present, but can be avoided by ensuring that the subject does not tilt or move the plate or their head during testing.

The Frisby-Davis distance (FD-2) stereotest uses four small real objects presented inside an open fronted box, at 6 metres 

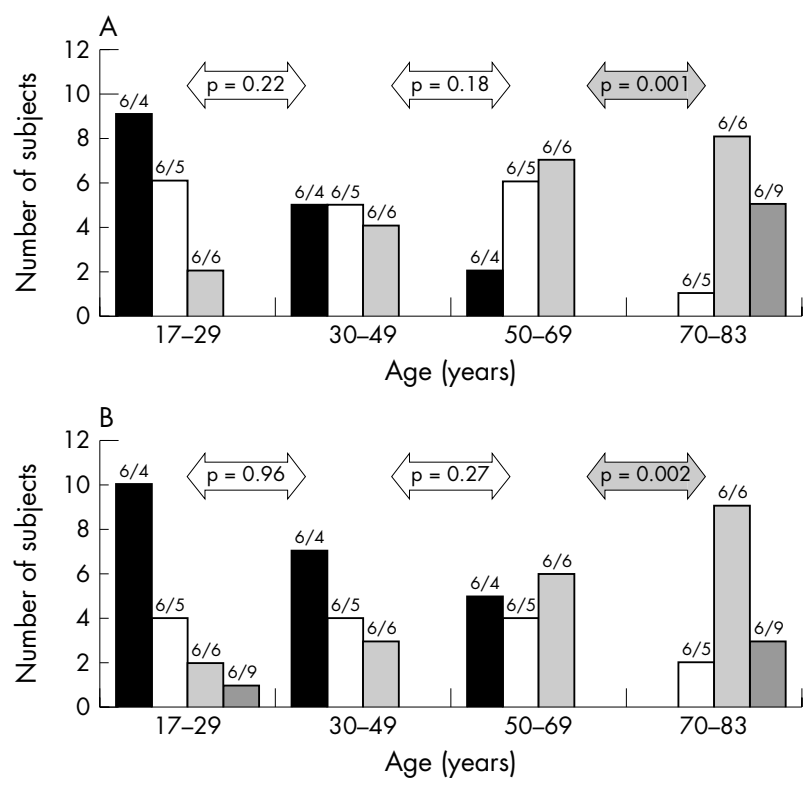

Figure 1 Histograms showing distribution of Snellen acuities for right eyes (A) and left eyes (B). The $p$ values in each arrow represent the significance of the difference in logMAR equivalent acuities between the adjacent groups calculated by unpaired $t$ tests.

from the subject, and the threshold for detecting which of the four objects is at a different distance from the subject is measured. This is therefore a test of "real world" stereoacuity. Monocular parallax clues are present, so it important to ensure that subjects do not move their heads during testing.

\section{Statistical analysis}

Distance and near Snellen acuities were converted to logMAR equivalents and analysed overall in relation to age using linear regression. Unpaired $t$ tests were used for subsequent comparison of distance acuity between age groups (Excel, Office XP; Microsoft). Overall change of stereoacuity and
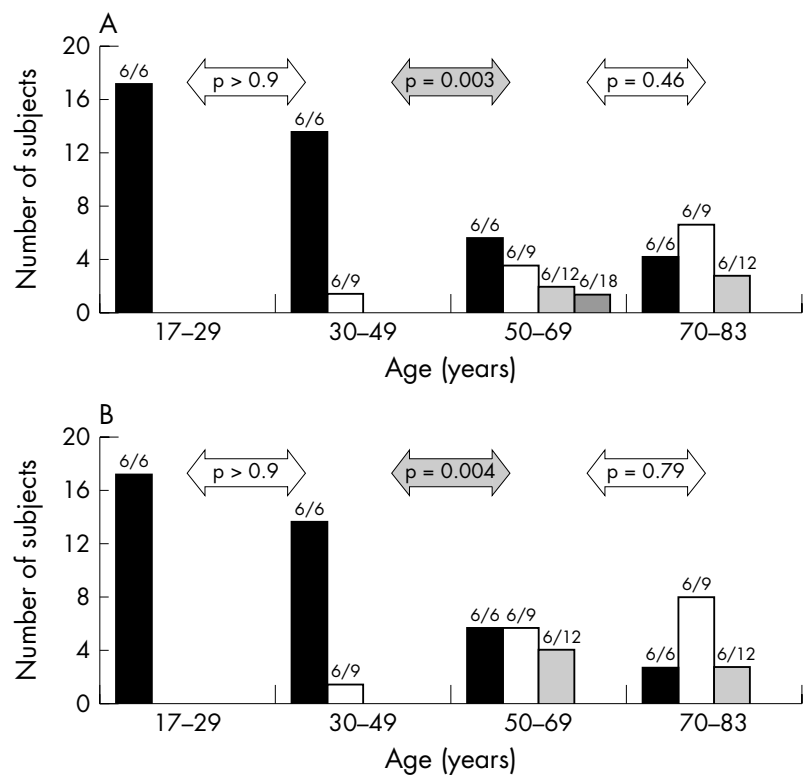

Figure 2 Histograms showing distribution of reduced Snellen acuities for right eyes (A) and left eyes (B). The p values in each arrow represent the significance of the difference in logMAR equivalent acuities between the adjacent groups calculated by the Mann-Whitney $U$ test. motor fusion with age were initially analysed using the Spearman rank correlation test corrected for tied values. The change in stereoacuity with age was then studied in more detail by dividing the subjects in four age bands; comparisons between different age groups for each stereotest were then made using the Mann-Whitney $U$ test corrected for tied values. This test was also used for near Snellen acuity because of the high number of tied values (Statview; SAS Institute Inc). Comparisons between stereoacuities measured in the same subject groups by different stereotests were made using the Wilcoxon signed rank test (Statview; SAS Institute Inc). The ratios of the stereoacuities measured by different stereotests were log transformed and analysed in relation to age by linear regression.

\section{RESULTS}

Distance Snellen acuity

Distance Snellen acuity showed a small overall reduction with age $\left(r^{2}=0.39\right.$ right; 0.24 left; $p<0.001$ for each eye;
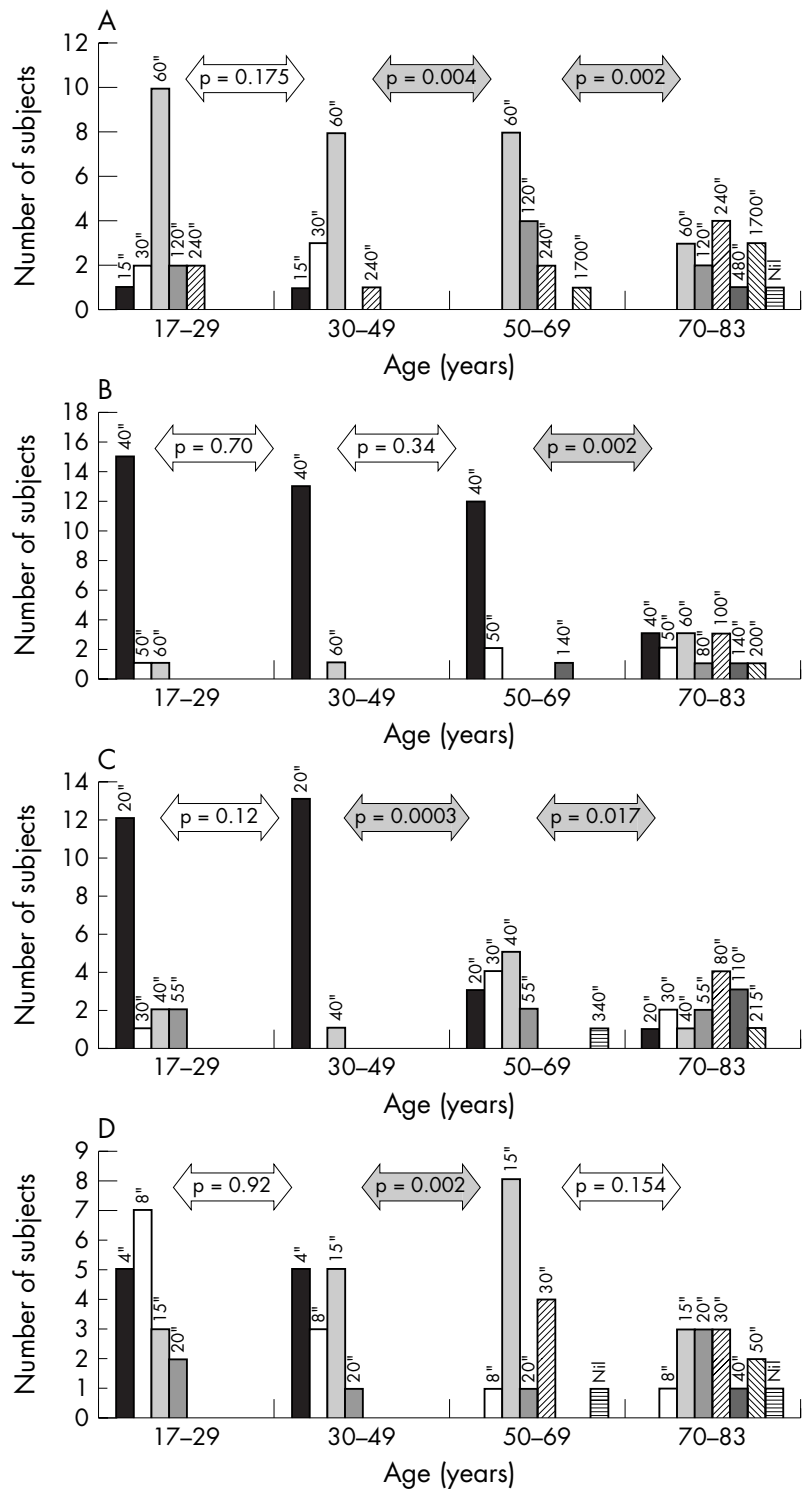

Figure 3 Histograms showing distribution of stereoacuities in 60 subjects divided into age group as measured by TNO (A), Titmus (B), Frisby near (C), and Frisby-Davis distance stereotests (D). The $p$ values in each arrow represent the significance of the difference in stereoacuity between the adjacent groups calculated by the Mann-Whitney $U$ test. 
linear regression of $\log$ MAR equivalent best corrected acuity, with left and right eyes analysed separately). Data from the subjects were then divided into groups, each covering an age span of approximately 20 years (fig 1 ). The main reduction in acuity was found to occur between the 50-69 year old and the 70-83 year old groups. Patients with a best corrected acuity of less that $6 / 9$ in either eye had been excluded, which may have lead to an underestimation of this age change.

\section{Near Snellen acuity}

The most significant reduction in near Snellen acuity occurred between the 30-49 and 50-69 year old groups (fig 2).

\section{Stereoacuity}

Overall, stereoacuity as measured by all four stereotests declined with age $(\mathrm{p}<0.001$ for all stereotests; Spearman rank correlation. Rho; TNO 0.55, Titmus 0.56, Frisby near 0.62 , Frisby distance 0.61 ), with the change being most marked for the TNO test.

For more detailed analysis of the relation between age and stereoacuity, the data were then analysed by comparing age bands, 17-29, 30-49, 50-69, and 70-83 years. For TNO and Frisby near stereoacuities there was a significant reduction between both the 30-49 and 50-69 age groups and between the 50-69 and 70-83 groups, whereas the Titmus test only showed a significant drop between the two latter age groups (fig 3). The Frisby-Davis distance stereoacuity showed a significant reduction between the 30-49 year old groups and the 50-69 year old group, with no significant later change (fig 3).

With the TNO stereotest five subjects, all aged 57 years or older, showed a markedly reduced stereoacuity. However, all these subjects were able to achieve at least 200 seconds of arc with the Titmus stereotest and 340 seconds of arc using the Frisby near stereotest (table 1). None of these subjects had an interocular acuity difference of more than one line in Snellen acuity and their reduced Snellen acuity was no worse than their peers (cf fig 2).

The data were further analysed by calculating the ratio of TNO to Titmus and TNO to Frisby near stereoacuities for each subject (fig 4). This ratio increased significantly with age (TNO/Titmus: $r^{2}=0.21 ; \mathrm{p}<0.001$; TNO/Frisby near: $r^{2}=0.08$; $\mathrm{p}=0.027$; linear regression of $\log$ ratio). It remained significant for the TNO/Titmus ratio when the five outliers described above were excluded $\left(r^{2} 0.09 ; \mathrm{p}=0.027\right)$, but not for the TNO/Frisby near ratio $\left(r^{2}=0.0002 ; p=0.91\right)$. These graphs confirm the selective loss of TNO in relation to Titmus and Frisby near stereoacuity in these five subjects.

\section{Comparison of stereotests}

Stereoacuity as measured for all subjects by the TNO test was significantly worse than for any of the other stereotests
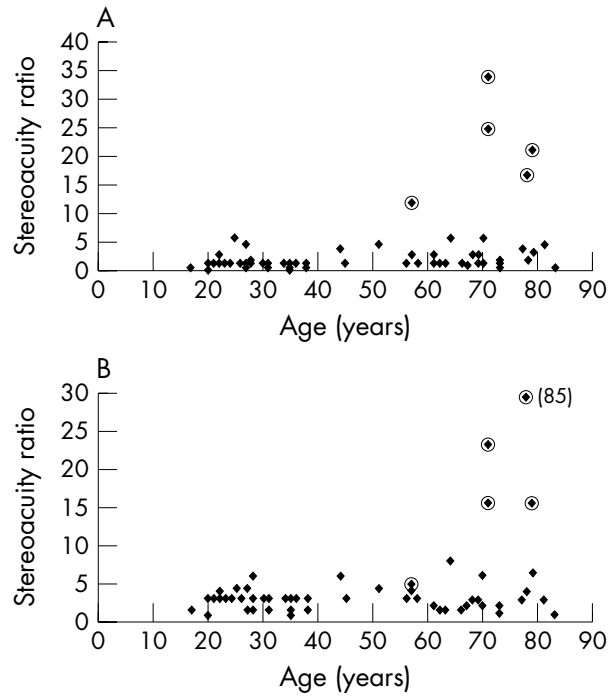

Figure 4 (A) The ratio of TNO stereoacuity divided by Titmus stereoacuity for the 60 subjects plotted against age. Note the five older subjects whose ratio is high (symbols in circles). (B) The ratio of TNO stereoacuity divided by Frisby near stereoacuity for the 60 subjects plotted against age. The same five older subjects are circled as in (A).

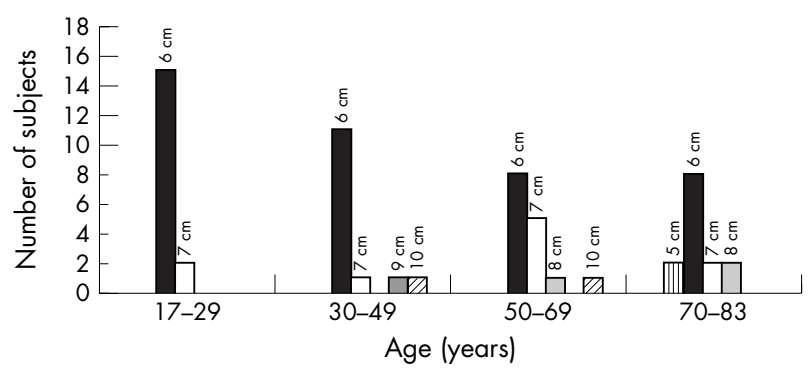

Figure 5 Histograms showing the distribution of the near point of convergence in 60 subjects divided into age groups. There was no significant trend in near point with age (Spearman rank correlation and linear regression).

(median values: TNO, 60 seconds of arc; Titmus, 40 seconds of arc; Frisby near, 30 seconds of arc; Frisby-Davis distance, 15 seconds of arc. TNO stereoacuity significantly different from each of the other tests, all $\mathrm{p}<0.001$, Wilcoxon signed rank test).

\section{Convergence}

There was no significant change in the near point of convergence with age (Rho 0.15; $\mathrm{p}=0.24$; Spearman rank correlation) (fig 5).

Table 1 Sensory data for five outliers with poor stereoacuity on TNO testing

\begin{tabular}{|c|c|c|c|c|c|c|c|c|}
\hline \multirow[b]{2}{*}{ Age (years) } & \multicolumn{2}{|c|}{ Snellen acuity $(6 \mathrm{~m})$} & \multicolumn{2}{|c|}{$\begin{array}{l}\text { Reduced Snellen } \\
\text { acuity }(1 / 3 \mathrm{~m})\end{array}$} & \multicolumn{4}{|c|}{ Stereoacuity (seconds of arc) } \\
\hline & Right & Left & Right & Left & TNO & Titmus & $\begin{array}{l}\text { Frisby } \\
\text { near }\end{array}$ & $\begin{array}{l}\text { Frisby } \\
\text { distance }\end{array}$ \\
\hline 57 & $6 / 6$ & $6 / 5$ & $6 / 6$ & $6 / 6$ & 1700 & 140 & 340 & $\mathrm{Nil}$ \\
\hline 71 & $6 / 6$ & $6 / 6$ & $6 / 6$ & $6 / 6$ & 1700 & 50 & 110 & 50 \\
\hline 71 & $6 / 9$ & $6 / 9$ & $6 / 12$ & $6 / 12$ & Nil & 200 & 215 & 50 \\
\hline 78 & $6 / 9$ & $6 / 6$ & $6 / 12$ & $6 / 9$ & 1700 & 100 & 20 & 30 \\
\hline 79 & $6 / 6$ & $6 / 9$ & $6 / 9$ & $6 / 9$ & 1700 & 80 & 110 & 15 \\
\hline Median & & & & & 1700 & 100 & 110 & 40 \\
\hline Median for other subjects aged 50 and over & & & & & 120 & 40 & 40 & 15 \\
\hline
\end{tabular}




\section{Motor fusion}

There was a small decline in the base out motor fusion range measured for distance fixation at 6 metres (Rho -0.26; $\mathrm{p}<0.05$; Spearman rank correlation), but no significant change in base in range for distance or either base out or base in range for near (fig 6). Combined normal values for motor fusion range are given in figure 7.

The five subjects with poor TNO stereoacuity above did not differ in motor fusion range from the mean of all other subjects aged over 50 (table 2 ).

\section{DISCUSSION}

In this study a single experienced observer has measured stereoacuity in a large group of patients across the whole adult age range using a battery of stereotests available in clinical practice. This has confirmed that there is some reduction in stereoacuity with age in subjects with normal Snellen acuity and no history of ophthalmic disease; it has also provided information as to when the reduction occurs. Although a number of studies have described reduced stereoacuity in elderly subjects ${ }^{1-10}$ most have not included younger adults for direct comparison, have not identified when the reduction occurs, and have used only one measure of stereoacuity.

The results for all four stereotests used in this study show that there is some decline of stereoacuity with age and that this affects both near and distance stereoacuity. It seems likely that this reflects a mild decline in the function of cortical disparity detectors with age as suggested from previous studies. However, five older patients in the present study showed a much greater reduction in stereoacuity, to screening or absent levels when tested with the TNO stereotest, than when measured by the other tests even though their Snellen distance and near acuities were no worse than other subjects in the same age groups. In a previous study a proportion of normal elderly subjects have been described as showing a "catastrophic" drop in stereoacuity using a short presentation of a random dot test ${ }^{10}$ and two population studies have also found a substantial proportion of elderly patients with poor stereoacuity. ${ }^{3}$

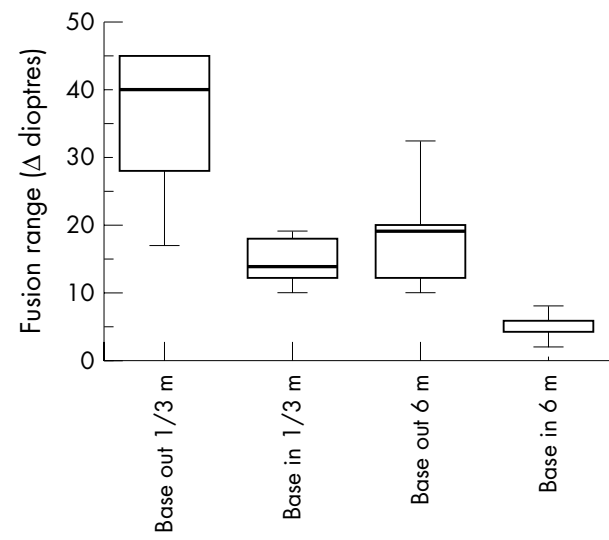

Figure 7 Overall motor fusion ranges for all 60 patients taken together.

However, these latter studies included patients with reduced vision from ophthalmic disease, which may have accounted for many of these. When Wright et al restricted their analysis to patients without other ophthalmic disease they still found a proportion of subjects with poor stereoacuity, defined as worse than 340 seconds of arc on the Frisby near test. The majority of these were over 75 years old, which may explain the greater proportion compared to the present study where only seven subjects were over 75 and all were fit enough to travel to the eye clinic. The present study confirms the finding that a substantial proportion of otherwise normal older subjects have poor stereoacuity when measured by a random dot stereotest. However, all these patients had much better stereoacuity when measured with the Titmus stereotest, which uses a contoured stimulus. This suggests that the reduction found in these elderly subjects using a random dot test may be caused as much by difficulties in performing this type of test as by a true loss of stereoacuity at cortical level.

Random dot stereotests, such as the TNO test, are generally considered the gold standard for measuring stereoacuity, because depth can only be perceived by the detection of the
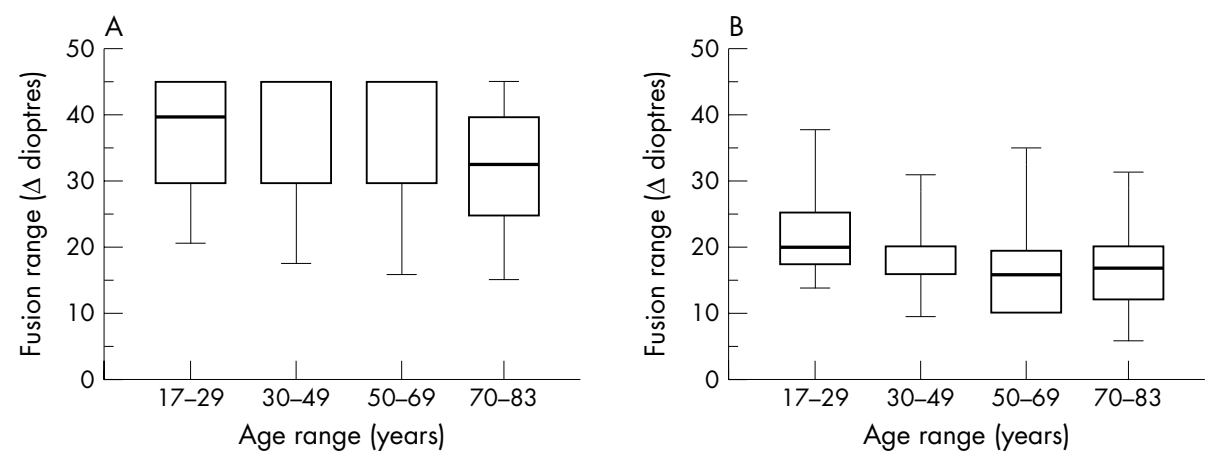

Figure 6 Motor fusion ranges for all 60 patients divided by age group. (A) Base out $1 / 3$ metre, (B) Base out 6 metres, (C) base in $1 / 3$ metre, and (D) base in 6 metres. Only the base out fusion range at 6 metres showed a significant decline with age ( $p<0.05$; Spearman rank correlation).
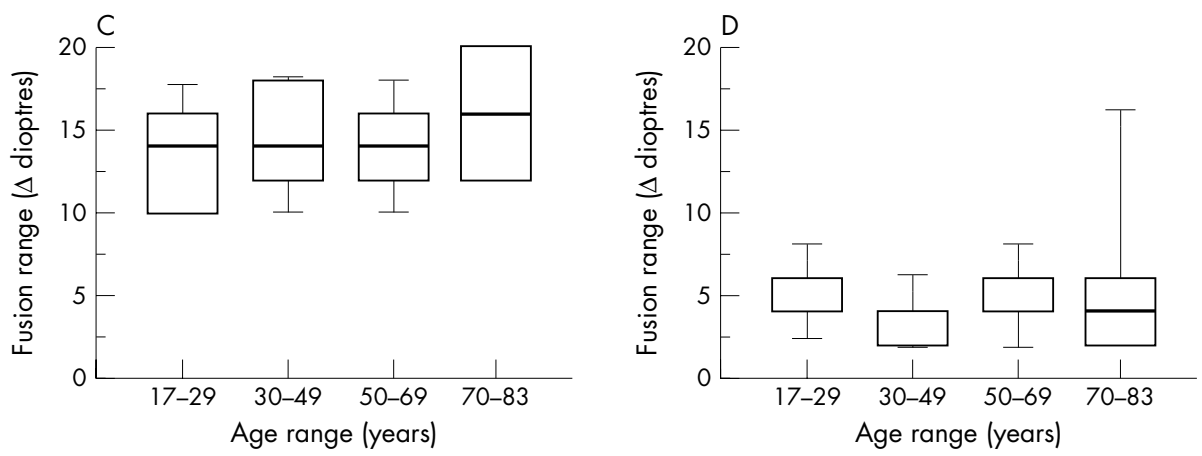
Table 2 Motor data for five outliers with poor stereoacuity on TNO testing

\begin{tabular}{|c|c|c|c|c|c|c|c|}
\hline \multirow[b]{2}{*}{ Age (years) } & \multicolumn{6}{|c|}{ Motor fusion range (prism dioptres) } & \multirow[b]{2}{*}{$\begin{array}{l}\text { Convergence } \\
(\mathrm{cm})\end{array}$} \\
\hline & $\begin{array}{l}\text { Base out } \\
1 / 3 \mathbf{~ m}\end{array}$ & $\begin{array}{l}\text { Base in } \\
1 / 3 \mathrm{~m}\end{array}$ & $\begin{array}{l}\text { Total } \\
1 / 3 \mathbf{~ m}\end{array}$ & $\begin{array}{l}\text { Base out } \\
6 \mathrm{~m}\end{array}$ & $\begin{array}{l}\text { Base in } \\
6 \mathrm{~m}\end{array}$ & $\begin{array}{l}\text { Total } \\
6 \mathrm{~m}\end{array}$ & \\
\hline 57 & 45 & 18 & 63 & 18 & 6 & 24 & 6 \\
\hline 71 & 40 & 20 & 60 & 20 & 16 & 36 & 6 \\
\hline 71 & 45 & 14 & 59 & 4 & 2 & 6 & 7 \\
\hline 78 & 40 & 14 & 54 & 20 & 4 & 24 & 6 \\
\hline 79 & 16 & 20 & 36 & 20 & 6 & 26 & 5 \\
\hline Mean & 37.2 & 17.2 & 54.4 & 16.4 & 6.8 & 23.2 & 6 \\
\hline $\begin{array}{l}\text { Mean for other } \\
\text { subjects aged } 50 \\
\text { and over }\end{array}$ & 34.2 & 14.6 & 48.8 & 17.7 & 4.9 & 22.6 & 6.6 \\
\hline
\end{tabular}

disparities between the sets of random dots presented to the two eyes and no monocular clues are present. However, recognition of the stereotarget requires a complex computation of correlation between the image elements presented to the two eyes and the generation of contours not present in the monocular stimuli. This also requires accurate motor alignment of the eyes in the absence of monocularly visible contours outlining the stereotarget. In contrast, the Titmus stereotest utilises real contours, with the disparate elements being presented using Polaroid glasses. Precautions were taken in the present study to ensure that the subjects were perceiving true depth with this test and not simply identifying one circle as different, in particular by inverting the test to reverse the disparity and checking that the stimulus changed from appearing in front of, to behind, the page. Fawcett and Birch ${ }^{12}$ have recently compared the Titmus test with two Randot tests in children with known abnormalities of binocular function and concluded that observers can have confidence in Titmus scores of 160 second of arc or better, even in children. The subjects in the present study were cooperative adults and most, including four of the five with poor random dot stereoacuity (table 1), had a Titmus stereoacuity of 140 seconds of arc or better. The differences discussed above probably also account for the substantially better levels of stereoacuity measured by the contour containing tests across the whole age range.

Standard clinical stereotests were used in this study rather than more prolonged psychophysical testing methods to allow rapid testing by several different methods without significant subject fatigue and to obtain data that could be directly related to clinical practice. This does lead to some significant limitations. The accuracy of thresholds determined was limited by the testing steps inherent in each test. In particular, the best stereoacuity measured by the Titmus test is 40 seconds of arc and this may have led to significant underestimation of contour based stereoacuity in younger subjects, and thus a possible reduction in stereoacuity at an earlier age than that demonstrated between the 50-69 and $70-83$ year old age groups could have been missed. It also means that the apparently better stereoacuities measured by the Frisby near and distant tests compared to the Titmus test may be artefactual. Conversely, the effect of the lack of real contours in the TNO test in comparison with the Titmus tests may be significantly greater than observed.

In the real world most judgments of depth are performed utilising the disparity of real contours in the objects of regard, which is more like the stimuli utilised by the Titmus stereotest than the TNO test. The large drop in stereoacuity seen in some older subjects using the TNO stereotest is probably because of a failure to overcome the dissociative effect of the test on fusion rather than a true loss of stereopsis at cortical level. The lack of subjective problems with depth perception noticed by normal subjects in the present study, in the report by Wright et al, ${ }^{3}$ and in patients with a unilateral macular hole who mostly retain stereopsis at screening levels ${ }^{11}$ would also indicate that subjects with only coarse stereopsis can function well in everyday life and that substantial problems with depth perception generally occur only when stereopsis is lost completely. In previously normal subjects, this is usually found in association with a marked reduction of vision in one eye. The functional benefit of second eye cataract surgery in restoring binocular function is well documented, ${ }^{13}$ but this may be as much the result of the restoration of gross as of fine stereopsis. Poor stereoacuity found utilising random dot stereotests alone should be interpreted with some caution, particularly in relation to everyday visual function in older patients.

\section{ACKNOWLEDGEMENTS}

The authors are grateful to professors John Frisby and Gary Rubin for their comments on the manuscript.

The study was approved by the hospital ethical committee and conformed to the tenets of the Declaration of Helsinki.

\section{Authors' affiliations}

L Garnham, J J Sloper, Moorfields Eye Hospital, City Road, London ECIV 2PD, UK

No competing interests.

Study conducted at Moorfields Eye Hospital, London, UK.

\section{REFERENCES}

1 Jani SN. The age factor in stereopsis screening. Am J Optom Arch Am Acad Optom 1966:43:653-7.

2 Greene HA, Madden DJ, Adult age differences in visual acuity, stereopsis, and contrast sensitivity. Am J Optom Physiol Opt 1987;64:749-53

3 Wright LA, Wormald RP. Stereopsis and ageing. Eye 1992;6:473-6.

4 Rumsey KE. Redefining the optometric examination: addressing the vision needs of older adults. Optom Vis Sci 1993;70:587-91.

5 Brown B, Yap MK, Fan WC. Decrease in stereoacuity in the seventh decade of life. Ophthalmic Physiol Opt 1993;13:138-42.

6 Yap M, Brown B, Clarke J. Reduction in stereoacuity with age and reduced retinal illuminance. Ophthalmic Physiol Opt 1994;14:298-301.

7 Rubin GS, West SK, Munoz B, et al. A comprehensive assessment of visual impairment in a population of older Americans. The SEE Study. Salisbury Eye Evaluation Project. Invest Ophthalmol Vis Sci 1997;38:557-68.

8 Haegerstrom-Portnoy G, Schneck ME, Brabyn JA. Seeing into old age: vision function beyond acuity. Optom Vis Sci 1999;76:141-58.

9 Schneck ME, Haegerstrom-Portnoy G, Lott LA, et al. Ocular contributions to age-related loss in coarse stereopsis. Optom Vis Sci 2000;77:531-6.

10 Zaroff CM, Knutelska M, Frumkes TE. Variation in stereoacuity: normative description, fixation disparity, and the roles of aging and gender. Invest Ophthalmol Vis Sci 2003;44:891-900.

11 Mireskandari K, Garnham L, Sheard R, et al. A prospective study of the effect of a unilateral macular hole on sensory and motor binocular function and recovery following successful surgery. $\mathrm{Br} J$ Ophthalmol 2004;88:1320-4.

12 Fawcett SL, Birch E. Validity of the Titmus and Randot circles tasks in children with known binocular vision disorders. J AAPOS 2003;7:333-8.

13 Laidlaw DA, Harrad RA, Hopper CD, et al. Randomised trial of effectiveness of second eye cataract surgery. Lancet 1998;352:925-9. 